\title{
Estrutura fatorial do questionário de adoção do óleo vegetal in natura de acréscimo na dieta infantil
}

\author{
Factorial structure of the questionnaire for the inclusion \\ of in natura vegetable oil in the diet of children
}

Haydée Serrão Lanzillotti ${ }^{1}$

Maria Elisa Barros ${ }^{1}$

Fernanda da Motta Afonso ${ }^{1}$

Roseane Moreira Sampaio Barbosa ${ }^{2}$

Cinthia de Carvalho Couto ${ }^{1}$

Fernanda Lopes Barreto ${ }^{1}$

${ }^{1}$ Instituto de Nutrição, Universidade do Estado do Rio de Janeiro. R. São Francisco Xavier 280, Maracanã. 20550-013 Rio de Janeiro RJ Brasil. haydeelan@gmail.com

${ }^{2}$ Universidade Federal

Fluminense. Niterói RJ Brasil.

\begin{abstract}
The scope of this study was to investigate the factorial structure of the questionnaire for the inclusion of vegetable oil in natura in the diet of children. A cross-sectional study was conducted with 220 mothers using the Likert Scale questionnaire. Thirteen Child Nutrition experts/judges semantically evaluated the items and the Generalizability Coefficient (GC) assessed the errors according to a one-off, two-facet random model (item/judge). The Exploratory Factor Analysis (EFA) indicated dimensionality of the tools applied: Doornik-Hansen Test; Main Factor Analysis; Kaiser Guttman criterion; Promax rotation; Kaiser-Meyer-Olkin test (KMO); convergent and discriminant validities, Cronbach's Alpha; Composite Reliability. The GC $=0.8407$ was considered satisfactory. The Doornik-Hansen test (chi2 $(28)=198.053$, prob $>$ chi2 $=0.0000)$ confirmed the multivariate normality of data. The Kaiser Guttman criterion explained $76.32 \%$ of the latent construct. The correlation between factors 1 and 2 (-0.4042) suggests the two-dimensional nature of the instrument. The KMO (0.8440) indicated the adequacy of the data to perform EFA. Cronbach's alpha showed values around 0.70, considered acceptable. The two-dimensionality of the questionnaire was verified to assess the inclusion of in natura vegetable oils in the diet of children.
\end{abstract}

Key words Child, Oil, Questionnaire, Factor analysis
Resumo $O$ objetivo foi verificar a estrutura fatorial do questionário de adoção do óleo vegetal in natura de acréscimo na dieta infantil. Estudo transversal, com 220 mães de crianças, e questionário em escala Likert. Treze juízes, especialistas em nutrição infantil, avaliaram semanticamente os itens, enquanto que o Coeficiente de Generalização (CG) mensurou erros de avaliação, segundo modelo randômico de duas facetas (item/juiz), uma ocasião. A Análise Fatorial Exploratória (AFE) indicou dimensionalidade do instrumento aplicado: teste Doornik-Hansen, Análise de Fator Principal, critério Kaiser Guttman, rotação Promax, teste Kaiser-MeyerOlkin (KMO), validades convergente e discriminante, coeficiente alfa de Cronbach, Confiabilidade Composta. O CG = 0,8407 foi considerado satisfatório. O teste Doornik-Hansen $\left(\mathrm{Qui}^{2}(28)=198,053\right.$, prob $>$ Qui $=$ 0,0000 ) inferiu sobre a normalidade multivariada dos dados. O critério de Kaiser Guttman indicou $76,32 \%$ da latência do construto. A correlação entre os fatores 1 e $2(-0,4042)$ sugere a bidimensionalidade do instrumento. KMO $(0,8740)$ indicou a adequação dos dados para AFE. O alfa de Cronbach apresentou valores em torno de 0,70, considerados aceitáveis. Verificou-se bidimensionalidade do questionário para avaliar a adoção da prática do acréscimo de óleos vegetais in natura na dieta infantil.

Palavras-chave Criança, Óleo, Questionário, Análise fatorial 


\section{Introdução}

As crianças constituem um dos grupos mais vulneráveis da população uma vez que estão expostas não só as adversidades de condições socioeconômicas como também ao entendimento que os pais/responsáveis pela alimentação da criança têm a respeito de conceitos técnicos científicos de uma alimentação saudável. A prevalência da desnutrição foi reduzida em cerca de 50\%: de 13,5\% (IC 95\%: 12,1\%; 14,8\%) em 1996 para 6,8\% $(5,4 \% ; 8,3 \%)$ em $2006 / 7^{1}$. Na tentativa de continuar esta redução, o Ministério da Saúde criou em 2012 a Agenda para Intensificação da Atenção Nutricional à Desnutrição Infantil ${ }^{2}$.

Dentre as diretrizes da Política de Alimentação e Nutrição do Brasil, aprovada por meio da Portaria MS/GM n. ${ }^{\circ} 710$, de 10 de junho de 1999, encontram-se a prevenção e o controle dos distúrbios nutricionais, bem como a promoção de práticas alimentares e estilos de vida saudáveis. Estas diretrizes, no tocante aos grupos populacionais de risco, estão sendo operacionalizadas através - Programa de Assistência Integral à Saúde da Mulher, Criança e Adolescente ${ }^{3}$.

O Ministério da Saúde, na publicação "Dez passos para uma alimentação saudável”, recomenda o acréscimo de óleo vegetal in natura nas refeições para aumentar a densidade energética da dieta ${ }^{4}$. O óleo vegetal com este intuito deverá estar imune de peróxidos, ou seja, deverá ser utilizado in natura, uma vez que quando aquecido no processo de fritura, possibilita uma série de reações químicas como hidrólise, oxidação e polimerização do óleo, produzindo numerosos compostos de degradação. Neste sentido, os óleos vegetais poliinsaturados, incluindo o óleo de soja (ácidos graxos: 15,2\% saturado, 23,3\% mono-insaturado e $60,0 \%$ polinsaturado), apresentam alterações em suas cadeias químicas quando submetidos à altas temperatura. O óleo de soja é o mais acessível ao consumo ${ }^{5}$ devido ao custo mais baixo.

Os lactentes alimentados com leite humano recebem apreciáveis quantidades de ácido araquidônico (AA) e ácido docosahexaenóico (DHA), que participam das reações bioquímicas das funções cognitivas (sistema nervoso) e visuais ${ }^{6,7}$. No entanto, com a introdução da alimentação complementar, a partir dos seis meses ou até antes, sob a forma de papas de frutas e papa salgada de hortaliças e leguminosas, as crianças reduzem a quantidade de ingestão destes nutrientes, uma vez que irão substituir o leite materno, inicialmente por duas refeições e em seguida por quatro. Os óleos vegetais ainda contribuem para a modulação da resposta imunológica, que advêm de sua composição lipídica ${ }^{8}$. Estas são razões para o uso do óleo vegetal in natura, além de promover aumento da densidade energética da dieta.

No entanto, a literatura especializada também aponta para limitações fisiológicas dos óleos vegetais que podem provocar o esvaziamento gástrico mais lento e, como consequência, náusea e vomito9. Os autores ressaltam que a recomendação do acréscimo de óleos vegetais in natura seja associado com outros alimentos ${ }^{9,10}$ e que a quantidade de óleos vegetais comestíveis in natu$r a$, recomendada pelo guia alimentar para população brasileira ${ }^{4}$ está fundamentada no principio nutricional da moderação ${ }^{11}$.

Embora a recomendação do acréscimo de óleo vegetal in natura tenha sido proposta em $2002^{4}$, até a presente data não foi verificado se esta recomendação tornou-se uma prática dietética na recuperação da desnutrição infantil. Este cenário criou a necessidade de se elaborar um instrumento, pelo qual se poderia inferir se pais e responsáveis pela alimentação da criança adotariam tal prática a partir de seus conhecimentos sobre o uso do óleo vegetal in natura na dieta infantil. Não se pode deixar de reconhecer o bombardeio da mídia televisiva contra o consumo de lipídios sem diferenciar entre suas classes, dado que diferem entre si na estrutura química, sejam vegetal, animal e trans.

A partir da revisão da literatura que discorre sobre óleo vegetal, segundo o enfoque de sua composição nutricional e fisiologia na alimentação humana, identificou-se a possibilidade de se explicitar o construto "Adoção do óleo vegetal in natura de acréscimo na dieta infantil" e duas dimensões supostamente teóricas a considerar: "benefícios do acréscimo de óleos vegetais in natura na dieta infantil" e "possíveis problemas relacionados ao acréscimo de óleos vegetais in natura na dieta infantil".

O desafio foi flagrar o entendimento de pais e responsáveis, pela alimentação da criança, sobre um conjunto de conceitos em relação ao uso do óleo vegetal in natura na intervenção da desnutrição infantil. Para tanto, fundamentou-se em Wilson ${ }^{12}$, que define o construto como parte de um modelo teórico da cognição, no qual uma pessoa ou grupo de pessoas assimilam certo conjunto de conceitos e direcionam sua atitude à alguma coisa. Neste entendimento pode ser distinguidos níveis de qualidade entre os extremos de concordância aos itens propostos como variáveis (itens) mensuráveis do construto, assim, 
uma ideia latente existe ao invés de um comportamento manifesto.

A construção de um questionário que possa avaliar a predisposição dos pais e responsáveis pela alimentação da criança a adotar a prática recomentada pelo Ministério da Saúde ${ }^{4}$ justifica-se dado a necessidade de se avaliar o direcionamento, positivo ou negativo, em relação a tal prática.

$\mathrm{O}$ que antecede a aplicação de um questionário é verificar a composição dos construtos no nível das suas variáveis observáveis. Embora se reconheça a dificuldade de criar um instrumento novo de mensuração ${ }^{13}$, foi proposto um estudo exploratório, onde se inclui a identificação das dimensões que compõem o construto.

O objetivo geral do estudo foi verificar a estrutura fatorial do questionário de adoção do óleo vegetal in natura de acréscimo na dieta infantil. Para tanto, se fez necessário: a) identificar a dimensionalidade do questionário para avaliar a adoção da prática do acréscimo de óleos vegetais in natura na dieta infantil; b) verificar a confiabilidade do questionário para avaliar a adoção da prática do acréscimo de óleos vegetais in natura na dieta infantil, c) verificar a validade fatorial, convergente e discriminante do questionário para avaliar a adoção da prática do acréscimo de óleos vegetais in natura na dieta infantil.

\section{Método}

\section{Aspectos legais}

Este estudo faz parte do projeto de pesquisa "Óleo Vegetal Comestível na Dieta como Estratégia de Aumento da Densidade Energética" aprovado pelo Comitê de Ética em Pesquisa da Universidade do Estado do Rio de Janeiro (RJ). Os participantes, após serem esclarecidos sobre os objetivos da pesquisa assinaram o Temo de Consentimento Livre e Esclarecido.

\section{Grupo de interesse}

Estudo transversal, amostra foi do tipo acidental $^{14}$, realizado com 220 mães e/ou acompanhantes de crianças, as quais aguardavam a consulta na sala de espera do ambulatório de Pediatria da Policlínica Piquet Carneiro/UERJ e do hospital universitário Pedro Ernesto (HUPE/ UERJ), Rio de Janeiro, RJ, 2013.

Elegeu-se como critério de inclusão para a aplicação do questionário que os entrevistados fossem os responsáveis pela alimentação da crianca, seja comprando alimentos, preparando -os ou servindo suas refeições, uma vez que estas situações podem ser consideradas intervenções de caráter dietético e, ainda, que as crianças não ultrapassassem dois anos de idade, de acordo com o Guia Alimentar para crianças menores de 2 anos $^{4}$. A coleta de dados foi realizada por acadêmicos de Nutrição devidamente treinados.

\section{Desenho dos itens}

O instrumento de mensuração para avaliar o construto "Adoção do óleo vegetal in

natura de acréscimo na dieta infantil" foi um questionário em escala Likert de cinco pontos. As questões foram construídas com direção positiva recebendo escores em ordem decrescente, ou seja, 5 (concordo muito); 4 (concordo); 3 (nem concordo /nem discordo); 2 (discordo) e 1 (discordo muito). De forma inversa, foram atribuídos os escores as questões negativas ${ }^{15}$ (Quadro 1).

Em conformidade com a recomendação de Streiner e Norman ${ }^{15}$ os itens do questionário foram criados mantendo-se numero de caracteres entre 40 e 80, a exceção do i15-P, que apresenta grafia com número superior de caracteres, uma vez que a redução deste comprometeria pressupostos teóricos em relação à hidrólise dos óleos vegetais, quando submetidos à temperatura de degradação ${ }^{16}$.

\section{Espaço de desfecho}

Nesta fase, 13 juízes, especialistas em Nutrição Materno Infantil, após assinarem o TCLE, analisaram semanticamente os itens e apontaram os escores para cada item da escala Likert, avaliando se estes traduzem o construto "adoção do óleo vegetal in natura de acréscimo na dieta infantil". A mensuração do erro valeu-se do Coeficiente de Generalização (CG), proposto pela Teoria da Generalização (TG). O CG avalia o quanto o resultado da avaliação de juízes especialistas, sobre os itens de um questionário, pode ser generalizável para o escore médio (ideal) que teria sido atribuído ao item sob todas as possíveis condições aceitáveis de avaliação ${ }^{17}$. O CG é dado:

$\mathrm{G}=$ variância item / (variância absoluta + variância item),

onde:

Variância relativa $=$ variância item. juiz, erro/ numero de itens do questionário.

Variância absoluta $=$ (variância item. juiz, erro + variância do item) / número de itens do questionário. A variância relativa privilegia todas 
Quadro 1. Protótipo 1 do questionário para avaliar a adoção do óleo vegetal in natura (cru) de acréscimo na dieta infantil, Rio de Janeiro,RJ, 2013.

\begin{tabular}{|l|l|}
\hline Item & Protótipo 1 \\
\hline i1-N & Colocar óleo vegetal cru na comida da criança piora a situação da criança com baixo peso $(73)^{\star}$ \\
\hline i2-N & O óleo vegetal cru atrapalha a digestão da criança $(42)^{\star}$ \\
\hline i3-N & Colocar óleo vegetal cru na comida da criança causa náuseas $(50)^{\star}$ \\
\hline i4-P & Colocar óleo vegetal cru na comida da criança melhora a situação da criança com baixo peso $(75)^{\star}$ \\
\hline i5-N & O óleo vegetal cru deixa a comida ruim $(31)^{\star}$ \\
\hline i6-P & Usar óleo vegetal cru na comida da criança melhora a imunidade da criança $(61)^{\star}$ \\
\hline i7-N & Se eu acrescentar óleo vegetal cru na comida da criança, ela ficará obesa $(61)^{*}$ \\
\hline i8-N & Colocar óleo vegetal cru na comida da criança causa vômitos $(50)^{\star}$ \\
\hline i9-P & É bom para a criança colocar óleo vegetal cru na mamadeira $(48)^{\star}$ \\
\hline i10-P & Usar óleo vegetal cru na comida da criança melhora sua resistência a doenças transmissíveis $(78)^{*}$ \\
\hline i11-N & Colocar óleo vegetal cru na comida da criança causa doenças por ele estar cru $(64)^{\star}$ \\
\hline i12-N & O óleo vegetal cru é transgênico $(27)^{\star}$ \\
\hline i13-N & O acréscimo de óleo vegetal cru eleva o colesterol da criança $(51)^{\star}$ \\
\hline i14-N & O uso de óleo vegetal cru todos os dias provoca diarreia. $(46)^{*}$ \\
\hline i15-P & $\begin{array}{l}\text { O certo é não refogar os alimentos da criança e colocar o óleo vegetal cru depois da papa salgada } \\
\text { pronta }(85)^{*}\end{array}$ \\
\hline
\end{tabular}

i: item, P: positivo, N:negativo; ${ }^{\star}$ Número de caracteres de cada item.

as interações que influenciam a posição relativa do juiz e a variância absoluta avalia o valor da mensuração do questionário, estabelecendo um índice aceitável para considerar as assertivas validas $^{17}$. Neste estudo foi desenvolvido modelo randômico de duas facetas e uma ocasião $0^{17,18}$.

\section{Modelo de medida}

No presente estudo, aplicou-se o teste Doornik-Hansen para testar a hipótese nula da normalidade multivariada segundo o p valor $\mathfrak{E}$ 0,05 . Neste procedimento, as observações multivariadas são transformadas em valores padronizados em unidades do desvio padrão, assim a assimetria e a curtose univariada são combinadas para aproximação da estatística qui-quadrada ${ }^{19}$.

Uma vez que se desconhecia a dimensionalidade do construto "Adoção do óleo vegetal in natura de acréscimo na dieta infantil", a AFE detectou os fatores que representam as dimensões latentes do construto $^{20}$. A AFE analisa o padrão de correlações existentes entre os escores atribuídos aos itens e utiliza esses padrões de correlações para agrupar os itens em fatores, que traduzem variáveis não observadas (variáveis latentes), as quais se vão medir a partir dos escores atribuídos aos itens (variáveis observadas), formando a matriz de entrada dos escores dos itens. Na AFE, a extração de fatores foi realizada pelo método de Análise de Fator Principal (AFP), inicialmente sem limitação do numero de fatores a serem re- tidos, utilizando o estimador de razão de verossimilhança-likelihoodratio (LR) e em seguida com rotação obliqua Promax.

Duas análises orientaram a seleção de fatores: critério de Kaiser Guttman (fatores com autovalor $>1$ ) e a adequação teórica ${ }^{21}$.

A partir dos resultados da AFE foram inferidos como itens satisfatórios os que apresentaram cargas fatoriais $(\lambda)$ maior $0,40^{22}$. Foram considerados itens com cargas cruzadas aqueles com cargas fatoriais similares em dois ou mais fatores em um mesmo item e cuja diferença entre as cargas fosse menor do que $0,10^{22}$. A variância do erro (uniqueness) foi considerada adequada quando a magnitude apresentou-se menor ou igual a $0,50^{20}$. As comunalidades foram vistas em termos de proporção da variância explicada por fatores subjacentes ${ }^{21}$.

Ao inferir sobre a dimensionalidade do questionário, foi considerada a correlação entre os fatores. Se a correlação entre os fatores (dimensões) apresentar-se baixa $(<0,30)$, de direção positiva, indicaria a possibilidade de dimensões de significados diferentes. No caso da correlação entre os fatores ser negativa, sugere-se que o fator não seja retirado. Se a correlação entre os fatores apresentar-se baixa/positiva ou baixa/negativa indica que o conteúdo de alguns itens do questionário não são discriminadores ${ }^{23}$.

A estatística Kaiser-Meyer-Olkin (KMO) indicou a adequação dos dados para a realização da análise fatorial, obtida pela soma dos quadrados 
das cargas fatoriais, que pode ser considerada comum a todas as variáveis, ou seja, que pode ser atribuída a um fator comum, então: quanto mais próximo de 1 (unidade) melhor o resultado, ou seja, mais adequada é a amostra à aplicação da análise fatorial ${ }^{19}$.

A validade fatorial convergente foi avaliada pela raiz da variância media extraída (VME), a qual representa o percentual médio de variação explicada pelos itens referentes ao construto, variando entre 0 a 1 . Valores de $\mathrm{VME} \geq 0,50$ sugerem que os itens compartilham de uma elevada variância em comum $^{24}$. A validade fatorial discriminante (VFD) é dada pela raiz quadrada de VME. Se a raiz quadrada da VME de um fator for maior do que as correlações entre este e os demais fatores corrobora-se a referida validade ${ }^{20}$.

O coeficiente alfa de Cronbach verificou a consistência interna do questionário e de suas dimensões. Alfa de Cronbach superior a 0,70 foi considerado aceitável ${ }^{25-27}$. Utilizou-se o procedimento de bootstrap para estimar os intervalos de confiança do coeficiente alfa ${ }^{28}$. Em estudos exploratórios, Hair et al. ${ }^{20}$ aceitam limite inferior do intervalo de confiança de alfa de Cronbach com valor de 0,60 .

Considerando que o coeficiente alfa de Cronbach não indica a 'verdadeira' confiabilida$\mathrm{de}^{23}$, optou-se por calcular a confiabilidade composta (CC), dada por:

$$
\mathrm{CC}=\frac{\left(\Sigma_{i=1}^{i=k} \lambda_{i}\right)}{\left(\mathrm{E}_{i=1}^{i=k} \lambda_{i}\right)+\left(\mathrm{E}_{i=1}^{i=k} \delta_{i}\right)}
$$

Onde:

$\lambda_{i}$ são cargas fatoriais $\delta_{i}$ variância do erro para $k$ itens.

A confiabilidade composta varia de 0 a 1 sendo considerada satisfatória, quando $\geq 0,70^{23}$.
A matriz de entrada dos escores em escala Likert foi computada em planilha eletrônica Excel. Os parâmetros de mensuração do modelo de analise fatorial exploratória, pelo aplicativo STATA versão $12.0^{29}$ e SPSS (Statistical Package for the Social Sciences) versão 18.

\section{Resultados}

No primeiro procedimento os 13 juízes avaliaram o conteúdo dos itens do questionário (quadro1). O CG alcançou o valor de 0,8407 (LR teste vs. regressão linear: Qui2 $=64,03$, prob $>$ Qui2 $=$ $0,0000)$, resultados não apresentados em tabela.

O teste Doornik-Hansen para os 15 itens do questionário $(\mathrm{n}=220)$, atestou aderência à distribuição Normal Multivariada, pois a estatística teste qui-quadrado observado com 28 graus de liberdade apresentou valor de 198,05 que conduz ao $\mathrm{p}_{\text {valor }}$ de 0,0000 , isto é, aceita-se a hipótese nula da distribuição multivariada.

A AFP sem limitação do numero de fatores, mostrou, pelo critério de Kaiser Guttman, (Tabela 1), que o fator 1 (autovalor de 3,9053) e o fator 2 (autovalor 1,0661) $\left(\mathrm{Qui}^{2}(55)=756,490\right.$, prob $>$ Qui $^{2}=0,0000$ ) explicaram 76,32\% da latência do construto. Do fator 3 em diante os autovalores apresentaram-se muito aquém do autovalor 1,0 , o que infere sobre a bidimensionalidade do questionário.

Analisando-se os resultados da AFP constrangida a dois fatores, sem rotação (Tabela 2), verifica-se que no fator 1 todos os itens apresentaram cargas fatoriais acima de $0,40 \mathrm{e}$, fator 2 , as cargas fatoriais apresentaram valores menores do que 0,40 , exceto as dos itens i8-N e i10-P. Ressalta-se que as variâncias do erro estão acima do

Tabela 1. Autovalores e variância explicada pela Análise de Fator Principal do estudo exploratório do questionário com 15 itens (Protótipo 1).Rio de Janeiro, RJ, 2013.

\begin{tabular}{lcccc}
\hline \multicolumn{1}{c}{ Fator } & Autovalor & Diferença & Proporção da variância explicada & Cumulativo \\
\hline Fator 1 & 3,9053 & 2,8391 & 0,5995 & 0,5995 \\
Fator 2 & 1,0661 & 0,6071 & 0,1637 & 0,7632 \\
Fator 3 & 0,4590 & 0,0464 & 0,0705 & 0,8336 \\
Fator 4 & 0,4125 & 0,1117 & 0,0633 & 0,8969 \\
Fator 5 & 0,3008 & 0,1409 & 0,0462 & 0,9431 \\
Fator 6 & 0,1598 & 0,0671 & 0,0245 & 0,9676 \\
Fator 7 & 0,0926 & 0,0077 & 0,0142 & 0,9819 \\
Fator 8 & 0,0849 & 0,0536 & 0,0130 & 0,9949 \\
Fator 9 & 0,0313 & 0,0292 & 0,0048 & 0,9997 \\
Fator 10 & 0,0021 & 0,0023 & 0,0003 & 1,0000 \\
Fator 11 & $-0,0002$ & & $-0,0000$ & 1,0000 \\
\hline
\end{tabular}

LR teste: independente vs. saturado: $\operatorname{Qui}^{2}(55)=756,49$ Prob $>$ Qui ${ }^{2}=0,0000$; Cargas fatoriais (matriz padrão) e variância do erro. 
Tabela 2. Cargas fatoriais $(\lambda)$, variâncias do erro $(\delta)$, validade fatorial convergente (VME) e validade fatorial discriminante (raiz VME), correlação entre fatores, alfa de Cronbach e confiabilidade composta do questionário reduzido a 11 itens (Protótipo 2), após a Análise de Fator Principal (AFP) constrangido a dois fatores, sem e com rotação oblíqua Promax. Rio de Janeiro, RJ, 2013.

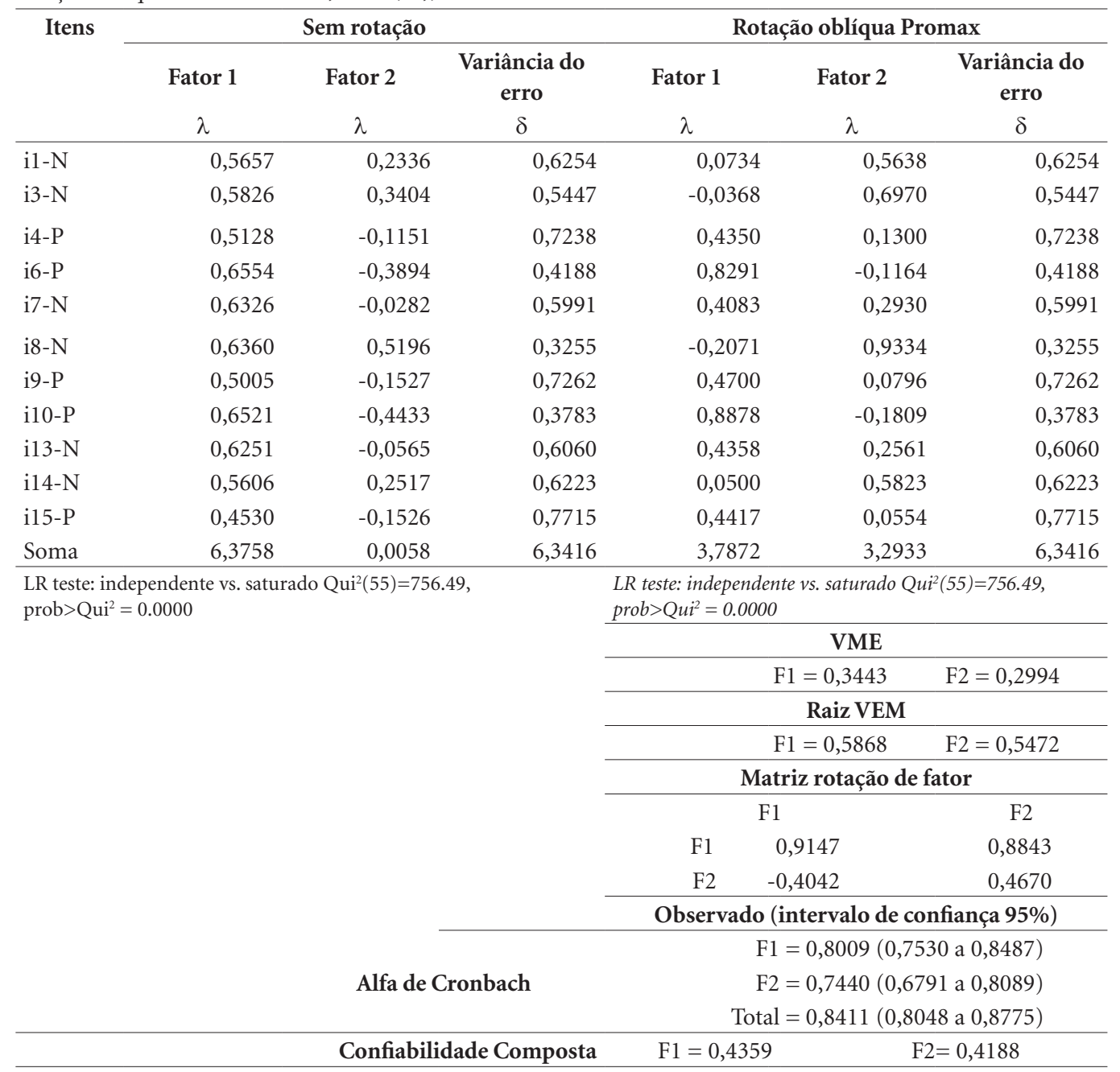

F1= Fator1, F2= Fator 2, VME= variância média extraída, LR = likelihood ratio. Fator 1: "possíveis problemas relacionados ao acréscimo de óleos vegetais in natura na dieta infantil". Fator 2: "benefícios do acréscimo de óleos vegetais in natura na dieta infantil”.

esperado (menor ou igual a 0,5 ), a exceção as dos itens i6-P, i8-N e i10-P. Este cenário infere sobre a unidimensionalidade do questionário. No entanto, analisando-se os resultados da AFP, constrangido a dois fatores com rotação obliqua Promax (Tabela 2), verificou-se distribuição dos itens, tanto no fator 1 quanto no fator 2, quando considerado cargas fatoriais acima de 0,40 . No fator 1 alocaram-se os itens i4-P, i6-P, i7-N, i9-P, i10-P, i13-N, i15-P e, no fator 2, os itens i1-N, i3-N, i8-N, i14-N. As variâncias do erro não apresentaram alteração. Estes resultados inferem sobre a bidimensionalidade do questionário, mostrando que a distribuição dos itens apresentou-se mais parcimoniosa.

A correlação entre os fatores 1 e 2 (Tabela 2), com valor $-0,4042$, corrobora a bidimensionalidade do instrumento que subjaz o questionário.

O teste KMO apresentou valor total de 0,8740 (dado não apresentado em tabela) indicando que o modelo de análise fatorial utilizado foi adequadamente ajustado aos dados.

No que se refere à confiabilidade, o alfa de Conbrach, para o fator 1 , fator 2 e para o total, apresentou valores em torno de 0,70 a luz do limite inferior (95\% confiabilidade), sendo consi- 
derada satisfatória. A confiabilidade composta, com valores de 0,4359 e 0,4188 , para os fatores 1 e 2 , respectivamente, apresenta-se aceitável ( $\mathrm{Ta}$ bela 2).

Em relação à validade fatorial, a convergente alcançou valores de 0,3443 e 0,2994 para os fatores 1 e 2, respectivamente. Estes valores não permitem afirmar que os itens compartilham de uma elevada variância em comum. A validade discriminante, com valores de 0,5868 (Fator 1) e 0,5472 (Fator 2) sugere o alcance desta, uma vez que a correlação entre o fatores 1 e 2 foi de $-0,4042$ (Tabela 2).

O conteúdo dos itens (Tabela 3) permitiu corroborar as dimensões teoricamente pressupostas, o fator 1 como "possíveis problemas relacionados ao acréscimo de óleos vegetais in natura na dieta infantil" e o fator 2 "benefícios do acréscimo de óleos vegetais in natura na dieta infantil", destoando os itens i7-N ("se eu acrescentar óleo vegetal cru na comida da criança, ela ficará obesa") e i13-N ("o acréscimo de óleo vegetal cru eleva o colesterol da criança"), por ter conteúdo negativo, no protótipo 1. Estes tiveram alteração semântica ("se eu acrescentar óleo vegetal cru na comida da criança, ela não ficará obesa") (i7-P) e ("o acréscimo de óleo vegetal cru não eleva o colesterol da criança") (i13-P), passando ambos a terem conteúdo positivo (Tabela 3). Os itens i4-P e i9-P apresentaram alto valor da variância de erro $(>0,70)$, mas permaneceram no questionário final (Tabela 3), devido aos pressupostos do Guia Alimentar para crianças menores de 2 anos. Os resultados permitiram construir o questionário final com 11 itens distribuídos em duas dimensões (Tabela 3).

\section{Discussão}

O presente estudo verificou a estrutura fatorial de um questionário que tem como escopo a possibilidade da adoção, por pais e responsáveis pela alimentação da criança, da prática do uso do óleo vegetal in natura de acréscimo na dieta infantil a fim de aumentar sua densidade energética.

Os procedimentos de elaboração de um instrumento novo em dietética, utilizando a AFE, permitiram identificar dois fatores: "possíveis problemas relacionados ao acréscimo de óleos vegetais in natura na dieta infantil" e "benefícios do acréscimo de óleos vegetais in natura na dieta infantil", fatores estes pressupostos teoricamente.

Publicações recentes de instrumentos de mensuração em saúde em geral são alvo de muita especulação na literatura, tanto no que se refe-

Tabela 3. Questionário final, bidimensional, a partir do modelo com rotação oblíqua Promax. Rio de Janeiro, RJ, 2013.

\begin{tabular}{|c|c|c|c|}
\hline $\begin{array}{l}\text { Itens - } \\
\text { fator } 1\end{array}$ & Descrição & $\begin{array}{l}\text { Itens - } \\
\text { fator } 2\end{array}$ & Descrição \\
\hline i4-P* & $\begin{array}{l}\text { Colocar óleo vegetal cru na comida da criança } \\
\text { melhora a situação da criança com baixo peso. }\end{array}$ & i1-N & $\begin{array}{l}\text { Colocar óleo vegetal cru na comida da } \\
\text { criança piora a situação da criança com } \\
\text { baixo peso. }\end{array}$ \\
\hline i6-P & $\begin{array}{l}\text { Usar óleo vegetal cru na comida da criança } \\
\text { melhora a imunidade da criança. }\end{array}$ & $\mathrm{i} 3-\mathrm{N}$ & $\begin{array}{l}\text { Colocar óleo vegetal cru na comida da } \\
\text { criança causa náuseas. }\end{array}$ \\
\hline $\mathrm{i} 7-\mathrm{P}^{\star \star}$ & $\begin{array}{l}\text { Se eu acrescentar óleo vegetal cru na comida } \\
\text { da criança, ela não ficará obesa. }\end{array}$ & $\mathrm{i} 8-\mathrm{N}$ & $\begin{array}{l}\text { Colocar óleo vegetal cru na comida da } \\
\text { criança causa vômito. }\end{array}$ \\
\hline i9- $\mathrm{P}^{*}$ & $\begin{array}{l}\text { É bom para a criança colocar óleo vegetal cru } \\
\text { na mamadeira. }\end{array}$ & $\mathrm{i} 14-\mathrm{N}$ & $\begin{array}{l}\text { O uso de óleo vegetal cru todos os dias } \\
\text { provoca diarreia. }\end{array}$ \\
\hline i10-P & $\begin{array}{l}\text { Usar óleo vegetal cru na comida da } \\
\text { criança melhora sua resistência a doenças } \\
\text { transmissíveis. }\end{array}$ & & \\
\hline i13-P** & $\begin{array}{l}\text { O acréscimo de óleo vegetal cru não eleva o } \\
\text { colesterol da criança. }\end{array}$ & & \\
\hline i15-P & $\begin{array}{l}\text { O certo é não refogar os alimentos da criança } \\
\text { e colocar o óleo vegetal cru depois da papa } \\
\text { salgada pronta. }\end{array}$ & & \\
\hline
\end{tabular}

Fator 1: "benefícios do acréscimo de óleos vegetais in natura na dieta infantil". Fator 2: "possíveis problemas relacionados ao acréscimo de óleos vegetais in natura na dieta infantil”. ${ }^{\star}$ Itens mantidos no questionário devido a plausibilidade teórica (ref Brasil. Ministério da Saúde. Dez passos para uma alimentação saudável. Guia Alimentar para crianças menores de 2 anos. Brasília, DF. 2002). ${ }^{* *}$ item com alteração semântica. 
re a sua dimensionalidade quanto a distribuição interna dos itens que o compõem. Neste estudo, foi utilizado procedimentos para examinar propriedades de mensuração em dietética, onde os estudos são raros. Uma exceção deste cenário foi o estudo realizado por Ling e Horwath ${ }^{30}$. Os autores utilizaram a análise de componentes principais, análise de fator confirmatória e índice de adequação de ajustamento do modelo a fim de desenvolver e validar uma escala de autoavaliação para discriminar indivíduos em estágios diferentes de disposição para mudança dos hábitos alimentares. Na fase exploratória do estudo, a análise de componentes principais revelou uma estrutura de dois fatores a qual foi responsável por $57 \%$ da variância na autoeficácia. Os dois fatores apresentaram alfa de Cronbach de 0,77 e 0,89 e cargas fatoriais que variam 0,59 a 0,86 .

Ressalta-se que, no presente estudo, foi elaborado um instrumento preliminar de mensuração sobre a prática de uso do óleo vegetal in natura de acréscimo. Por consequência, o mesmo está limitado à discussão de seus próprios resultados, uma vez que não foi encontrado na literatura instrumentos que discutam o construto proposto.

$\mathrm{Na}$ avaliação do questionário por juízes especialistas, CG alcançou valor expressivo de confiabilidade, ou seja, superou 0,70, conforme sugerido por Brown ${ }^{23}$. Este achado pode ser resultante da experiência profissional dos juízes especialistas em nutrição infantil e sua sintonia com as politicas publica de segurança nutricional e alimentar para a população infantil. O CG, neste contexto, difere de seu uso regular, ou seja, no desempenho escolar, e tornou possível abrir um espaço para a investigação de confiabilidade inferencial de instrumentos de avaliação em educação nutricional.

No que concerne à confiabilidade, o alfa de Cronbach atingiu valores satisfatórios nas duas dimensões que emergiram das análises e a confiabilidade composta mostrou-se baixa. A divergência entre o alfa de Cronbach e a confiabilidade composta sugere que sejam repensados os parâmetros de confiabilidade, quando se estuda a dimensionalidade de instrumentos de mensuração em saúde utilizando-se a análise fatorial. Enquanto o alfa de Cronbach alcançou valores em torno de $70 \%$, a confiabilidade composta indicou valores em torno de 0,40 , não obstante, Maroco $^{24}$ refere que valores abaixo de 0,70 são aceitáveis para investigações exploratórias. Autores ${ }^{23,31-33}$ têm afirmado a insuficiência do alfa de Cronbach para avaliar a confiabilidade. A proposta deste estudo foi uma iniciativa positiva, uma vez que demonstrou a forma de se avaliar a confiabilidade de um instrumento de mensuração em saúde utilizando-se análise estatística mais robusta. O mais importante foi mostrar que sem um estudo mais elaborado a determinação da dimensionalidade e da confiabilidade de um questionário pode se apresentar equivocada.

No que tange a validade fatorial, embora o instrumento apresente validade fatorial discriminante, não alcançou validade fatorial convergente nas dimensões, ou seja, itens não compartilharam de uma elevada variância em comum ${ }^{29}$, como pôde ser visto pelas variâncias do erro.

Em relação aos aspectos semânticos pertinentes aos itens, originalmente, i7- $\mathrm{N}$ ("se eu acrescentar óleo vegetal cru na comida da criança, ela ficará obesa”) e i13-N ("O acréscimo de óleo vegetal cru eleva o colesterol da criança"), foram reescritos de forma que assumissem conteúdos de direção positiva, ou seja, i7-P "se eu acrescentar óleo vegetal cru na comida da criança, ela não ficará obesa" e i13-P "O acréscimo do óleo vegetal cru não eleva o colesterol da criança”. Com relação à semântica usada no i7 ainda com conteúdo de direção negativa, seria verdade a consequência de obesidade na criança pelo acréscimo de óleo vegetal se não fossem obedecidas quantidades recomendadas pelo Ministério da Saude ${ }^{4}$, o que reforça que a aquiescência a tal recomendação seja feita por profissionais da saúde. Analogamente, no que se refere ao i13, a opção por alteração semântica pode ser justificada pela crença do senso comum, que os óleos, assim como as gorduras, elevam o colesterol sérico. Cabe ressaltar que os responsáveis pela alimentação da criança, possivelmente, não fazem diferença entre óleos e outras classes de lipídios. E mais, os participantes deste estudo, provavelmente, não têm uma compreensão holística a respeito dos benefícios do óleo vegetal in natura; aparentemente seriam considerados possíveis problemas relacionados a esta prática, dado que eles carecem de conhecimentos científicos sobre a composição dos alimentos.

Se existe uma recomendação do Ministério da Saúde e esta não é aquilatada por instrumentos pertinentes, ao se propor um questionário, mesmo que preliminar, se avança para verificar se o conteúdo da recomendação é alcançado pelas mães, pais e responsáveis pela alimentação da criança, atores sociais responsáveis pelo sucesso ou fracasso da adoção da prática do óleo vegetal in natura, de acréscimo na dieta infantil.

A principal limitação deste estudo refere-se à população de interesse, que foi restrita a dois ambulatórios universitários de pediatria da cida- 
de do Rio do Janeiro (RJ) e, por consequência, a validade externa não poderia ser alcançada.

A relevância do estudo foi apresentar tais procedimentos metodológicos para construir um questionário novo mostrando sua estrutura fatorial, evidências de confiabilidade e de validade e pressupostos teóricos necessários a sua construção.

\section{Conclusão}

Os procedimentos de construção do questionário para avaliar a adoção da prática do acréscimo de óleos vegetais in natura na dieta infantil, instrumento novo de mensuração dietética, possibilitaram mostrar a bidimensionalidade do questionário, confiabilidade plausível e validade fatorial discriminante satisfatória, no entanto, a convergente assumiu valores que não permitiram afirmar que os itens compartilham de uma elevada variância em comum. É um instrumento habilitado para o procedimento de Analise Fatorial Confirmatória em etapa posterior. Este estudo pode ser visto como um caminho ao desenvolvimento de instrumentos de mensuração em saúde como subsídios para intervenção nutricional.

\section{Colaboradores}

HS Lanzillotti participou na concepção, delineamento, análise dos dados e redação do artigo. ME Barros participou na análise dos dados e redação do artigo. FM Afonso participou da análise dos dados e na aprovação a ser publicada. RMS Barbosa participou da redação final e na aprovação a ser publicada. FL Barreto participou na pesquisa e na metodologia. CC Couto participou na pesquisa e na metodologia. 


\section{Referências}

1. Monteiro CA, Benicio MHD, Konno SC, Feldenheimer ACS, Lima ALL, Conde WL. Causas do declínio da desnutrição infantil no Brasil, 1996-2007. Rev Saúde Pública 2009; 43(1):35-44.

2. Brasil. Ministério da Saúde (MS). Manual instrutivo para implementação da Agenda para Intensificação da Atenção Nutricional à Desnutrição Infantil. Brasília: MS; 2013.

3. Brasil. Ministério da Saúde (MS). Política Nacional de Alimentação e Nutrição. Brasília: MS; 2013.

4. Brasil. Ministério da Saúde (MS). Dez passos para uma alimentação saudável: Guia Alimentar para crianças menores de 2 anos. Brasília: MS; 2002.

5. Nunes SP. Produção e consumo de óleos vegetais no Brasil. DESER [Boletim eletrônico]. 2007. [acessado 2016 maio 23]; 159. Disponível em: http://www.deser. org.br/documentos/doc/

6. Koletzko B. Ácidos graxos poliinsaturados de cadeia longa (LCPUFA) na dieta de lactentes prématuros. In: Seminário Nestlé Nutrition. Nutrition Workshop Series. 1998; Vevey, Suíça. p.6-9.

7. Sant'Ana LS. Biodisponibilidade dos lipídeos. In: Cozzolino SMF, organizador. Biodisponibilidade de nutrientes. Barueri: Manole; 2005. p. 152-170.

8. Calder PC. The effect of dietary fatty acids on the immune response and susceptibility to infection. In: Suskind RM, Tontisirin K, editors. Nutrition, Inmunity, and Infection in Infants and Children. Philadelphia: Nestlé Nutrition Workshop Series, Pediatric Program; 2001.

9. Mahan LK, Escott-Stump SK, Raymond JL. Alimentos, nutrição e dietoterapia. 9a ed. São Paulo: Roca; 1998.

10. Aires MM. Fisiologia. $3^{\text {a }}$ ed. Rio de Janeiro: Guanabara Koogan; 2008.

11. Barbosa RMS, Colares LGT, Soares EA. Guia alimentar para crianças de 2 e 3 anos. Rio de Janeiro: RUBIO; 2013.

12. Wilson M. Constructing Measure: an item response modeling Approach. London: Lawrence Erlbaum Associates; 2005.

13. Reichenheim ME, Morais C L. Qualidade dos instrumentos epidemiológicos. In: Almeida-Filho N, Barreto M, organizadores. Epidemiologia: Fundamentos, Métodos e Aplicação. Rio de Janeiro: Guanabara-Koogan; 2007. p. 150-159.

14. Selltiz C, Jahoda M, Deutsch M, Cook S. Métodos de pesquisa nas relações sociais. $3^{a}$ ed. São Paulo: EPU; 1974.

15. Streiner DL, Norman GR. Health measurement scales: A practical guide to their development and use. $4^{\text {th }} \mathrm{ed}$. Oxford: Oxford University Press; 2008.

16. Sanibal EAA, Mancini-Filho J. Alterações físicas, químicas e nutricionais de óleos submetidos ao processo de fritura. Food Ingred South Am 2002; 18:64-71.

17. Shavelson RJ, Webb NM. MMSS Generalizability Theory: A primer. Newbury Park, London: SAGE Publications; 1991.

18. Suen HK, Lei PW. Classical versus Generalizability theory of measurement. Educational Measurement 2007; 4:1-13.

19. Doornik JA, Hansen H. An omnibus test for univariate and multivariate normality. Oxford Bulletin of Economics and Statistics 2008; 70(Supl. 1):927-939.
20. Hair JF, Black WC, Babin BJ, Anderson RE, Tatham RL. SEM: confirmatory factor analysis. $6^{\text {th }}$ ed. Porto Alegre: Bookman; 2009.

21. Damasio BF. Uso da análise factorial exploratória em psicologia. Aval Psicol 2012; 11(2):213-228.

22. Bonfim CB, Santos DN, Menezes IG, Reichenheim ME, Barreto ML. Um estudo sobre a validade de construto da Parent-Child Conflict Tactics Scale (CTSPC) em uma amostra populacional urbana do Nordeste brasileiro. Cad Saúde Pública 2011; 27(11):2215-2226.

23. Brown TA. Confirmatory factor analysis for applied research. New York: The Guilford Press; 2006.

24. Maroco J. Análise de equações estruturais: Fundamentos teóricos, software \& aplicações. Pêro Pinheiro: Report Number; 2010.

25. Nunnaly JC. Psychometric theory. New York: McGraw -Hill; 1978.

26. Pasquali L. Psicometria: Teoria dos testes na Psicologia e na Educação. Petrópolis: Vozes; 2009.

27. Sijtsma, K. Reability beyond theory and into practice. Psychometrika 2009; 74(1):169-173.

28. Raykov T, Shrout PE. Reliability of Scales With General Structure: Point and Interval Estimation Using a Structural Equation Modeling Approach. Structural Equation Modeling: A Multidisciplinary Journal 2002; 9(2):195-212

29. Stata Corp LP, College Station [Computer program]. Version 12: Stata Structural Equation Modeling Reference: manual release 12. College Station: Stata Press Publication; 1985-2011.

30. Ling AM, Horwath C. Self-efficacy and consumption of fruit and vegetables: validation of a summated scale. Am J Health Promot 1999; 13(5):290-298.

31. Reichenheim ME, Moraes CL, Oliveira ASD, Lobato G. Revisiting the dimensional structure of the Edinburgh Postnatal Depression Scale (EPDS):empirical evidence for a general factor. BMC Med Res Methodol 2011; 11:93.

32. Castro MMLD, Hökerberg YHM, Passos SRL. Validade dimensional do instrumento de qualidade de vida WHOQOL-BREF aplicado a trabalhadores de saúde Cad Saúde Pública 2013; 29(7):1357-1369.

33. Maroco J, Oliveira RA, Vicente CS. Análise Fatorial do Inventário de Burnout de Maslach (mbi-hss) em Profissionais Portugueses. Psic Saúde \& Doenças 2013; 14(1):152-167.

Artigo apresentado em 08/03/2016

Aprovado em 22/04/2017

Versão final apresentada em 24/04/2017 